\title{
Improving Position Estimation Accuracy of Magnetic Saliency Based Sensorless Control by Considering Cross-Coupling Factor
}

\author{
Shinya Morimoto*a) Senior Member, \\ Masanobu Kakihara* Member, \\ Keita Shimamoto** \\ Member \\ Tsuyoshi Hanamoto*** Member
}

(Manuscript received Jan. 28, 2020, revised May 27, 2020)

J-STAGE Advance published date : Oct. 1, 2020

\begin{abstract}
This paper presents a method for improving the position estimation accuracy for magnetic saliency based sensorless control. Conventional magnetic saliency based sensorless position estimation methods are based on the voltage equation of Interior Permanent Magnet Synchronous Motor without incorporation of the mutual inductances between the $\mathrm{d}$-axis and q-axis of rotating reference frame synchronized with rotor. The mutual inductances are caused by magnetic saturation due to load current and high-frequency injection signal for position detection. Furthermore, the mutual inductances contain the harmonic components due to the motor structure. Consequently, the position estimation error is caused by the neglected mutual inductances. It is difficult to determine the actual value of mutual inductance, particularly the harmonic components that vary depending on the rotor position. Therefore, in this paper, a method for improvement of the position estimation accuracy by considering the cross-coupling factor without determining the mutual inductances is proposed. The experimental results demonstrate the validity of the proposed method.
\end{abstract}

Keywords: position sensorless, high frequency signal injection, estimation accuracy, cross-coupling factor

\section{Introduction}

Interior Permanent Magnet Synchronous Motor (IPMSM) have become popular in industrial applications. In order to utilize Field Oriented Control (FOC) for the IPMSM drive, the accurate rotor position information is essential. Thus, position sensors such as optical encoders or resolvers are usually employed. Removing position sensors have some advantages in sensor cable saving, higher torque density, and higher robustness to machine vibration. Therefore, position estimation method without position sensors has attracted wide attention ${ }^{(1)}$.

Various position estimation methods have been developed. Suitable methods for motor startup and low-speed operation are based on the magnetic saliency. The rotor-position is estimated from the current variation caused by the applied voltage. The typical methods are indirect flux detection by online reactance measurement method, high frequency voltage signal injection methods, and modified Pulse Width Modulation (PWM) methods ${ }^{(2)-(5)}$. As the algorithms are different, the frequency and waveform of applied voltage are different, respectively. In order to improve the sensorless control performance, position estimation algorithms have following two

a) Correspondence to: Shinya Morimoto. E-mail: Shinya.Mori moto@yaskawa.co.jp

${ }^{*}$ Corporate R\&D Center, YASKAWA Electric Corporation 12-1, Otemachi, Kokurakita-ku, Kitakyushu 803-8530, Japan

** Tsukuba Research Laboratory, YASKAWA Electric Corporation

5-9-10, Tokodai, Tsukuba, Ibaraki 300-2635, Japan

${ }^{* * *}$ Graduate School of Life Science and Systems Engineering, Kyushu Institute of Technology

2-4, Hibikino, Wakamatsu-ku, Kitakyushu 808-0196, Japan problems to be solved.

1: A time delay of estimation

2: Estimation errors

One of the solutions to the first problem is that the higher frequency voltage signal is used since estimation period can be shorter. In high frequency injection methods, the frequency of injected voltage signal needs to be close to the carrier frequency ${ }^{(6)}$. In modified PWM method, the vector pattern is modified using space vector PWM (SVPWM) to amplify the current variation during the carrier period ${ }^{(7)}$. As a result, estimation period of both high frequency injection methods and modified PWM methods can be close to the carrier period.

One solution to the second problem is to compensate for estimation errors using the characteristic information of the target IPMSM measured in advance. A Look-Up Table (LUT) of the position estimation error in every operation state of the target IPMSM is created by a preliminary test, and during actual operation, the estimated position is compensated by the data read from the LUT based on the operating state ${ }^{(8)(9)}$. Another solution is accounting for crosscoupling factor of IPMSM. The cross-coupling factor depends on motor design and current, and is known to be caused by magnetic saturation ${ }^{(10)(11)}$. Generally, the estimation algorithms are based on voltage equation without incorporating mutual inductances in dq-axis. According to conventional method, position estimation error is caused by neglecting the mutual inductances in dq-axis. However, it is difficult to determine the cross-coupling factor from mutual inductances in dq-axis. One of the methods to determine the factor is finite-element analysis. Estimation algorithms accounting for cross-coupling factor determined by finite-element analysis 
show improvement in the position estimation accuracy ${ }^{(12)(13)}$. However, these methods can compensate for cross-coupling factor due to magnetic saturation, but do not discuss slot harmonic components depending on the motor structure. Since the cross-coupling factor due to the slot harmonic changes depending on the rotor position, it causes a harmonic components in the position estimation error, which causes vibration and noise when the motor rotates, even if there is no load. In addition, data collection before the running of equipment is not practical in many industrial applications. Therefore, position estimation method accounting for cross-coupling factor without analytical values or data collection is required.

In this paper, a position estimation accuracy improvement method that can compensate for the slot harmonics of the motor is proposed. In this method, mutual inductances are used in the voltage equation to accounting for cross-coupling factor. In the proposed algorithm, the cross-coupling factor from mutual inductances is not required to determine. The dq-axis cross-coupling component is extracted from the applied voltage for position estimation and its response current, and the estimated position is compensated using the extracted components. In order to compare the estimation accuracy, the proposed method is applied to a modified PWM method described in reference (7). Experimental results demonstrate the effectiveness of the proposed method especially for slot harmonics.

\section{Influence of Dq-axis Mutual Inductances on Position Estimation}

Figure 1 shows the coordinates of IPMSM. The $\alpha-\beta$ frame is fixed to the stator winding. The $\alpha$-axis coincides with the $u$-phase direction. The $d-q$ frame expresses the rotating reference frame and the $d$-axis coincides with the $\mathrm{N}$-pole. The terminology used for parameters in this paper are shown in Table 1. The general voltage equation of IPMSM on the $d$ - $q$ frame is expressed as equation (1).

$$
\left[\begin{array}{l}
v_{d} \\
v_{q}
\end{array}\right]=\left[\begin{array}{cc}
R & -\omega L_{q} \\
\omega L_{d} & R
\end{array}\right]\left[\begin{array}{c}
i_{d} \\
i_{q}
\end{array}\right]+\left[\begin{array}{cc}
L_{d} & 0 \\
0 & L_{q}
\end{array}\right] \frac{d}{d t}\left[\begin{array}{l}
i_{d} \\
i_{q}
\end{array}\right]+\left[\begin{array}{c}
0 \\
\omega \psi
\end{array}\right]
$$
(2).

Transforming equation (1) on the $\alpha-\beta$ frame gives equation

$$
\begin{array}{r}
{\left[\begin{array}{l}
v_{\alpha} \\
v_{\beta}
\end{array}\right]=R_{a}\left[\begin{array}{l}
i_{\alpha} \\
i_{\beta}
\end{array}\right]+\left[\begin{array}{cc}
L+l \cos 2 \theta & l \sin 2 \theta \\
l \sin 2 \theta & L-l \cos 2 \theta
\end{array}\right] \frac{d}{d t}\left[\begin{array}{l}
i_{\alpha} \\
i_{\beta}
\end{array}\right]} \\
+2 l \omega\left[\begin{array}{cr}
-\sin 2 \theta & \cos 2 \theta \\
\cos 2 \theta & \sin 2 \theta
\end{array}\right]\left[\begin{array}{c}
i_{\alpha} \\
i_{\beta}
\end{array}\right]+\omega \psi\left[\begin{array}{c}
-\sin \theta \\
\cos \theta
\end{array}\right] \\
\ldots \ldots \ldots \ldots \ldots \ldots
\end{array}
$$

Considering only the high-frequency signal-injection components, equation (2) can be approximated as follows.

$$
\left[\begin{array}{c}
v_{\alpha h} \\
v_{\beta h}
\end{array}\right] \cong\left[\begin{array}{cc}
L+l \cos 2 \theta & l \sin 2 \theta \\
l \sin 2 \theta & L-l \cos 2 \theta
\end{array}\right] \frac{d}{d t}\left[\begin{array}{c}
i_{\alpha h} \\
i_{\beta h}
\end{array}\right] \ldots \ldots
$$

Generally, in a high-frequency injection sensorless method based on a fixed coordinate system, $\sin 2 \theta$ and $\cos 2 \theta$ components are extracted from several injection signal vectors and

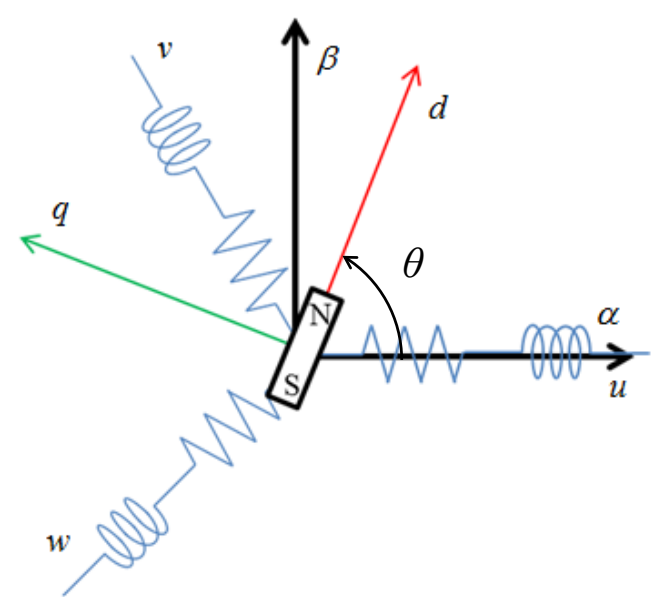

Fig. 1. Coordinate of IPMSM

Table 1. Terminology for parameters

\begin{tabular}{|l|l|l|l|}
\hline$v$ & Voltage & $\alpha, \beta$ & Fixed system coordinates \\
\hline$i$ & Current & $d, q$ & Rotated coordinates \\
\hline$R$ & Resistance & $u, v, w$ & Three phase \\
\hline$L, l$ & Inductance & $x$ & Mutual inductance component \\
\hline$\omega$ & Angular speed & res & Response \\
\hline$\theta$ & Position & $r e f$ & Reference \\
\hline$\Psi$ & Flux linkage & est & Estimation component \\
\hline$\zeta$ & Duty & conv & Conventional method \\
\hline $\mathrm{V}$ & Voltage vector & prop & Proposed method \\
\hline $\begin{array}{l}A, A^{\prime} \\
B, C, \\
K\end{array}$ & Arbitrary coefficient & $h$ & High frequency component \\
\cline { 3 - 4 } & & $\mathrm{V}_{\mathrm{a},} \mathrm{V}_{\mathrm{b}}$ & Effective voltage vectors \\
\hline
\end{tabular}

their response signals based on equation $(3)^{(4)-(7)}$. The generalized expressions are shown in equations (4) and (5).

$$
\begin{aligned}
& f_{\sin }\left(\frac{d i_{\alpha h}}{d t}, \frac{d i_{\beta h}}{d t}\right)=A \sin 2 \theta \\
& f_{\cos }\left(\frac{d i_{\alpha h}}{d t}, \frac{d i_{\beta h}}{d t}\right)=A \cos 2 \theta
\end{aligned}
$$

The coefficient $A$ is related to the injected voltage and the inductances. In the conventional method, the estimated position is obtained as in equation (6) from equations (4) and (5).

$$
\theta_{\text {conv }}^{e s t}=\frac{1}{2} \tan ^{-1}\left(\frac{f_{\sin }\left(\frac{d i_{\alpha h}}{d t}, \frac{d i_{\beta h}}{d t}\right)}{f_{\cos }\left(\frac{d i_{\alpha h}}{d t}, \frac{d i_{\beta h}}{d t}\right)}\right)
$$

On the other hand, the voltage equation of IPMSM accounting for dq-axis mutual inductances is expressed as equation (7) on the $d-q$ frame.

$$
\left[\begin{array}{l}
v_{d} \\
v_{q}
\end{array}\right]=\left[\begin{array}{cc}
R & -\omega L_{q} \\
\omega L_{d} & R
\end{array}\right]\left[\begin{array}{l}
i_{d} \\
i_{q}
\end{array}\right]+\left[\begin{array}{cc}
L_{d} & L_{d q} \\
L_{q d} & L_{q}
\end{array}\right] \frac{d}{d t}\left[\begin{array}{l}
i_{d} \\
i_{q}
\end{array}\right]+\left[\begin{array}{c}
0 \\
\omega \psi
\end{array}\right]
$$

By transforming equation (7) on the $\alpha-\beta$ frame and extracting only the high-frequency signal-injection components, an approximate equation (8) is obtained as follows. 


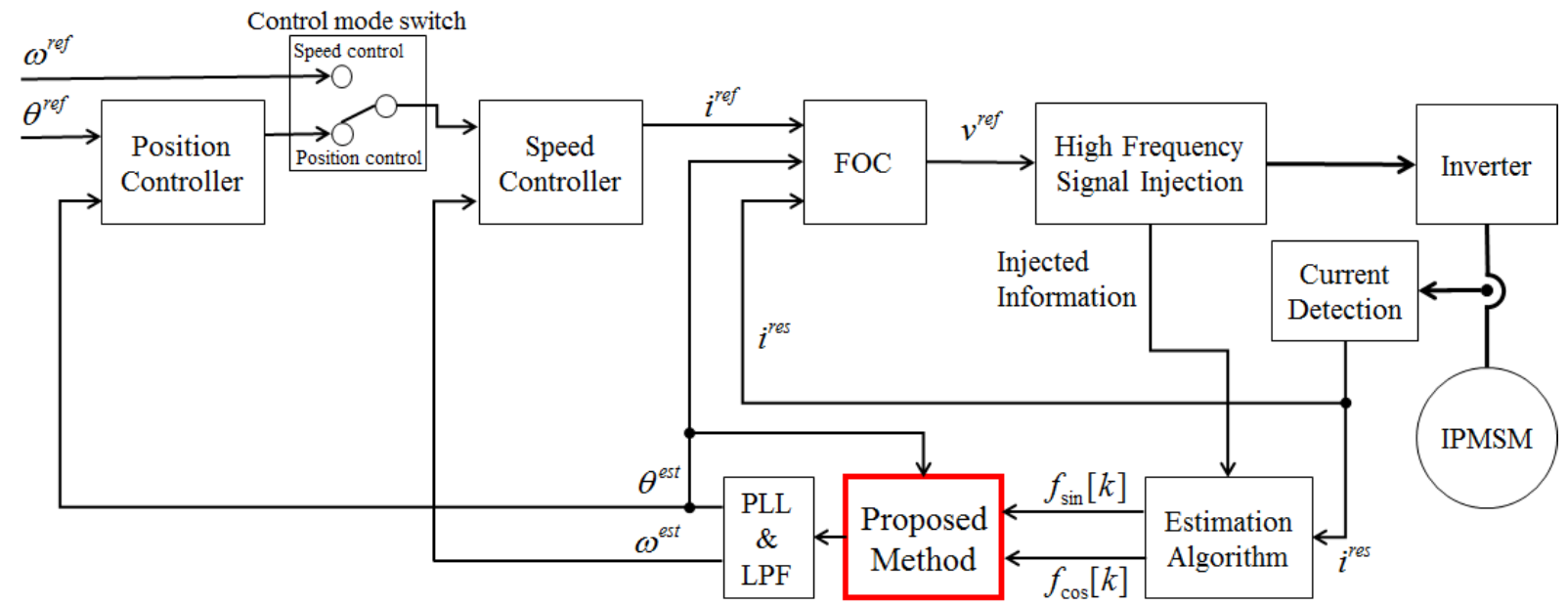

Fig. 2. Block diagram of control system with proposed method

$\left[\begin{array}{l}v_{\alpha h} \\ v_{\beta h}\end{array}\right] \cong\left[\begin{array}{ll}L(1,1) & L(1,2) \\ L(2,1) & L(2,2)\end{array}\right] \frac{d}{d t}\left[\begin{array}{l}i_{\alpha h} \\ i_{\beta h}\end{array}\right]$

$L(1,1)=L+l \cos 2 \theta-L_{x} \sin 2 \theta$

$L(1,2)=l \sin 2 \theta+l_{x}+L_{x} \cos 2 \theta$

$L(2,1)=l \sin 2 \theta-l_{x}+L_{x} \cos 2 \theta$

$L(2,2)=L-l \cos 2 \theta-L_{x} \sin 2 \theta$

$L_{x}=\frac{L_{d q}+L_{q d}}{2}, \quad l_{x}=\frac{L_{q d}-L_{d q}}{2}$

If dq-axis mutual inductances are not neglected, the sine and cosine functions $\left(f_{\sin }, f_{\text {cos }}\right)$ can be represented as equation (9) and equation (10).

$$
\begin{aligned}
& f_{\sin }\left(\frac{d i_{\alpha h}}{d t}, \frac{d i_{\beta h}}{d t}\right)=A^{\prime}\left(l \sin 2 \theta+L_{x} \cos 2 \theta\right) \\
& f_{\cos }\left(\frac{d i_{\alpha h}}{d t}, \frac{d i_{\beta h}}{d t}\right)=A^{\prime}\left(l \cos 2 \theta-L_{x} \sin 2 \theta\right)
\end{aligned}
$$

Equations (9) and (10) are rearranged as follows.

$$
\begin{aligned}
& {\left[\begin{array}{r}
f_{\sin }\left(\frac{d i_{\alpha h}}{d t}, \frac{d i_{\beta h}}{d t}\right) \\
f_{\cos }\left(\frac{d i_{\alpha h}}{d t}, \frac{d i_{\beta h}}{d t}\right)
\end{array}\right]=A^{\prime}\left[\begin{array}{cc}
\cos 2 \theta & \sin 2 \theta \\
-\sin 2 \theta & \cos 2 \theta
\end{array}\right]\left[\begin{array}{c}
L_{x} \\
l
\end{array}\right]} \\
& \ldots \ldots \ldots \ldots \ldots \ldots \\
& {\left[\begin{array}{cc}
\cos 2 \theta & -\sin 2 \theta \\
\sin 2 \theta & \cos 2 \theta
\end{array}\right]\left[\begin{array}{l}
f_{\sin }\left(\frac{d i_{\alpha h}}{d t}, \frac{d i_{\beta h}}{d t}\right) \\
f_{\cos }\left(\frac{d i_{\alpha h}}{d t}, \frac{d i_{\beta h}}{d t}\right)
\end{array}\right]=A^{\prime}\left[\begin{array}{c}
L_{x} \\
l
\end{array}\right]}
\end{aligned}
$$

From equation (12), equation (13) can be derived.

$$
\frac{f_{\sin }\left(\frac{d i_{\alpha h}}{d t}, \frac{d i_{\beta h}}{d t}\right) \cos 2 \theta-f_{\cos }\left(\frac{d i_{\alpha h}}{d t}, \frac{d i_{\beta h}}{d t}\right) \sin 2 \theta}{f_{\sin }\left(\frac{d i_{\alpha h}}{d t}, \frac{d i_{\beta h}}{d t}\right) \sin 2 \theta+f_{\cos }\left(\frac{d i_{\alpha h}}{d t}, \frac{d i_{\beta h}}{d t}\right) \cos 2 \theta}=\frac{L_{x}}{l}
$$

Furthermore, equations (9) and (10) can be rewritten as follows.

$$
\left[\begin{array}{c}
f_{\sin }\left(\frac{d i_{\alpha h}}{d t}, \frac{d i_{\beta h}}{d t}\right) \\
f_{\cos }\left(\frac{d i_{\alpha h}}{d t}, \frac{d i_{\beta h}}{d t}\right)
\end{array}\right]=A^{\prime} l\left[\begin{array}{cc}
1 & \frac{L_{x}}{l} \\
-\frac{L_{x}}{l} & 1
\end{array}\right]\left[\begin{array}{l}
\sin 2 \theta \\
\cos 2 \theta
\end{array}\right] \ldots
$$

From equation (14), equation (15) is obtained.

$$
\left[\begin{array}{c}
\sin 2 \theta \\
\cos 2 \theta
\end{array}\right]=\frac{1}{A^{\prime} l\left(1+\left(\frac{L_{x}}{l}\right)^{2}\right)}\left[\begin{array}{cc}
1 & -\frac{L_{x}}{l} \\
\frac{L_{x}}{l} & 1
\end{array}\right]\left[\begin{array}{l}
f_{\sin }\left(\frac{d i_{\alpha h}}{d t}, \frac{d i_{\beta h}}{d t}\right) \\
f_{\cos }\left(\frac{d i_{\alpha h}}{d t}, \frac{d i_{\beta h}}{d t}\right)
\end{array}\right]
$$

Therefore, estimated position from proposed method is expressed as equation (16). Since $L_{x} / l$ is obtained by equation (13), position can be estimated.

$$
\begin{aligned}
2 \theta_{\text {prop }}^{\text {est }} & =\tan ^{-1}\left(\frac{\sin 2 \theta}{\cos 2 \theta}\right) \\
& =\tan ^{-1}\left(\frac{f_{\sin }\left(\frac{d i_{\alpha h}}{d t}, \frac{d i_{\beta h}}{d t}\right)-\frac{L_{x}}{l} f_{\cos }\left(\frac{d i_{\alpha h}}{d t}, \frac{d i_{\beta h}}{d t}\right)}{\frac{L_{x}}{l} f_{\sin }\left(\frac{d i_{\alpha h}}{d t}, \frac{d i_{\beta h}}{d t}\right)+f_{\cos }\left(\frac{d i_{\alpha h}}{d t}, \frac{d i_{\beta h}}{d t}\right)}\right)
\end{aligned}
$$

Here, by transforming equation (16) using the arctangent addition formula and substituting equation (6), equation (17) is obtained.

$$
\begin{aligned}
\theta_{\text {prop }}^{\text {est }} & =\frac{1}{2} \tan ^{-1}\left(\frac{f_{\sin }\left(\frac{d i_{\alpha h}}{d t}, \frac{d i_{\beta h}}{d t}\right)}{f_{\cos }\left(\frac{d i_{\alpha h}}{d t}, \frac{d i_{\beta h}}{d t}\right)}\right)-\frac{1}{2} \tan ^{-1}\left(\frac{L_{x}}{l}\right) \\
& =\theta_{\text {conv }}^{\text {est }}-\frac{1}{2} \tan ^{-1}\left(\frac{L_{x}}{l}\right) \ldots \ldots \ldots \ldots \ldots \ldots \ldots
\end{aligned}
$$

Therefore, the position estimation error $\Delta \theta$ due to the dqaxis mutual inductances in the conventional method can be expressed as follows.

$$
\Delta \theta=\frac{1}{2} \tan ^{-1}\left(\frac{L_{d q}+L_{q d}}{L_{d}-L_{q}}\right) .
$$




\section{Proposed Method in Discrete Time}

By obtaining $L_{x} / l$ from equation (13), it is possible to estimate the position using equation (16) or (17) while eliminating the influence of mutual inductances. However, in the actual operation of equation (13) cannot be obtained, since actual position is unknown. Therefore, last estimated position is utilized for the multiplication. It is assumed that the difference between the actual position and the last estimated position is negligibly small, since the sampling time is very small. The assumption is expressed by equation (19).

$$
\theta[k] \approx \theta^{e s t}[k-1] .
$$

Therefore, equations (13) can be rewritten as follows.

$$
\frac{f_{\text {sin }}[k] \cos 2 \theta_{\text {prop }}^{\text {est }}[k-1]-f_{\cos }[k] \sin 2 \theta_{\text {prop }}^{\text {est }}[k-1]}{f_{\text {sin }}[k] \sin 2 \theta_{\text {prop }}^{\text {est }}[k-1]+f_{\cos }[k] \cos 2 \theta_{\text {prop }}^{\text {est }}[k-1]} \approx \frac{L_{x}}{l}
$$

As a result, $L_{x} / l$ and estimated position can be obtained from the same algorithm expressed by equations (13). Moreover, as the estimated position is calculated from current information, the estimation accuracy depends on the accuracy of the current information. That is, the estimated position becomes noisy due to presence of noise in current signal. In that case, the assumption might have bad effect on the position estimation. Therefore, the sensitivity parameter " $a$ " is defined. By using the sensitivity parameter, estimated position can be rewritten as equation (21).

$$
2 \theta_{\text {prop }}^{e s t}[k]=\tan ^{-1}\left(\frac{f_{\sin }[k]-\frac{a L_{x}}{l} f_{\cos }[k]}{\frac{a L_{x}}{l} f_{\sin }[k]+f_{\cos }[k]}\right) \ldots \ldots \ldots
$$

When the sensitivity parameter " $a$ " is 0 , equation (21) is the same as the conventional method.

Block diagram of sensorless control system with proposed method is shown in Fig. 2 that shows that no additional information is required for the proposed method. $f_{\sin }[k]$ and $f_{\text {cos }}[k]$ are output of conventional position estimation methods. Therefore, the proposed method is easily attached to the conventional position estimation methods based on voltage equation expressed by equation (2).

\section{Application to the Modified PWM Method}

This section shows an estimation algorithm of the modified PWM method with accounting for dq-axis inductances as an example. The applied method is described in reference (7). This method uses modified SVPWM to amplify the current variation. High frequency component of equation (8) is expressed in equation (22), with the determinant $B$ of the inductance matrix.

$$
\frac{d}{d t}\left[\begin{array}{c}
i_{\alpha h} \\
i_{\beta h}
\end{array}\right]=\frac{1}{B}\left[\begin{array}{cc}
L(2,2) & -L(1,2) \\
-L(2,1) & L(1,1)
\end{array}\right]\left[\begin{array}{c}
v_{\alpha h} \\
v_{\beta h}
\end{array}\right] \ldots \ldots \ldots
$$

Figure 3 shows the conceptual diagram of SVPWM. $V_{a}$ and $V_{b}$ are vector notations of efficient voltage vectors. $V_{a}$ represents $\mathrm{V}_{1}, \mathrm{~V}_{2}$, and $\mathrm{V}_{4}$, whereas $\mathrm{V}_{\mathrm{b}}$ represents $\mathrm{V}_{3}, \mathrm{~V}_{5}$, and $\mathrm{V}_{6}$. Equation (22) with $\mathrm{V}_{\mathrm{a}}$ is expressed as (23), and (22) with $V_{b}$ is expressed as (24).

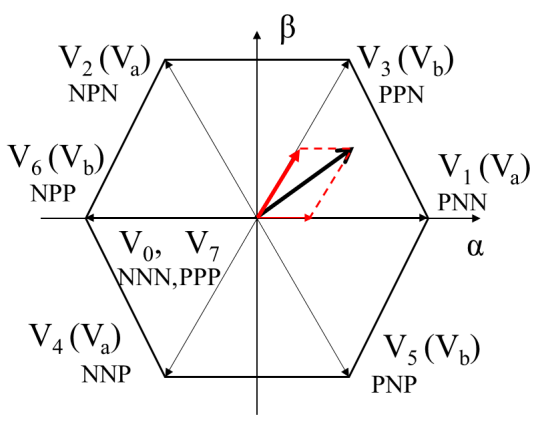

Fig. 3. Space vector PWM

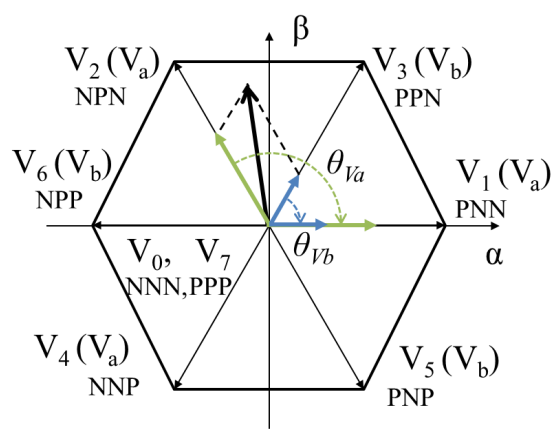

Fig. 4. Rotational coordinate transformation

$$
\begin{aligned}
& \frac{d}{d t}\left[\begin{array}{l}
i_{\alpha h \mathrm{Va}} \\
i_{\beta h \mathrm{Va}}
\end{array}\right]=\frac{1}{B}\left[\begin{array}{cc}
L(2,2) & -L(1,2) \\
-L(2,1) & L(1,1)
\end{array}\right]\left[\begin{array}{c}
v_{\alpha h \mathrm{Va}} \\
v_{\beta h \mathrm{Va}}
\end{array}\right] \ldots \ldots \\
& \frac{d}{d t}\left[\begin{array}{c}
i_{\alpha h \mathrm{Vb}} \\
i_{\beta h \mathrm{Vb}}
\end{array}\right]=\frac{1}{B}\left[\begin{array}{cc}
L(2,2) & -L(1,2) \\
-L(2,1) & L(1,1)
\end{array}\right]\left[\begin{array}{l}
v_{\alpha h \mathrm{Vb}} \\
v_{\beta h \mathrm{Vb}}
\end{array}\right] \ldots \ldots
\end{aligned}
$$

In order to correspond to the direction of the output voltage vector with $\alpha$-axis, rotational coordinate transformation is used. The transformation is rotation by the angle of the output voltage vector as shown in Fig. 4. As a result, the voltage vector component of $\beta$-axis direction is zero. Therefore, equations (25) and (26) can be obtained.

$$
\begin{aligned}
& \frac{d}{d t}\left[\begin{array}{l}
i_{\alpha h r \mathrm{Va}} \\
i_{\beta h r \mathrm{aa}}
\end{array}\right]=\frac{v_{\alpha h r \mathrm{Va}}}{B}\left[\begin{array}{l}
L_{r \mathrm{Va}}(1,1) \\
L_{r \mathrm{Va}}(2,1)
\end{array}\right] \ldots \ldots \ldots \ldots \ldots(25) \\
& L_{r \mathrm{Va}}(1,1)=L-l \cos \left(2 \theta-2 \theta_{\mathrm{Va}}\right)+L_{x} \sin \left(2 \theta-2 \theta_{\mathrm{Va}}\right) \\
& L_{r \mathrm{Va}}(2,1)=-l \sin \left(2 \theta-2 \theta_{\mathrm{Va}}\right)-l_{x}-L_{x} \cos \left(2 \theta-2 \theta_{\mathrm{Va}}\right) \\
& \frac{d}{d t}\left[\begin{array}{l}
i_{\alpha h r \mathrm{Vb}} \\
i_{\beta h r \mathrm{Vb}}
\end{array}\right]=\frac{v_{\alpha h r \mathrm{Vb}}}{B}\left[\begin{array}{l}
L_{r \mathrm{Vb}}(1,1) \\
L_{r \mathrm{Vb}}(2,1)
\end{array}\right] \ldots \ldots \ldots \ldots(26) \\
& L_{r \mathrm{Vb}}(1,1)=L-l \cos \left(2 \theta-2 \theta_{\mathrm{Vb}}\right)+L_{x} \sin \left(2 \theta-2 \theta_{\mathrm{Vb}}\right) \\
& L_{r \mathrm{Vb}}(2,1)=-l \sin \left(2 \theta-2 \theta_{\mathrm{Vb}}\right)-l_{x}-L_{x} \cos \left(2 \theta-2 \theta_{\mathrm{Vb}}\right)
\end{aligned}
$$

Assuming that the magnitude $\mathrm{V}_{\mathrm{a}}$ and $\mathrm{V}_{\mathrm{b}}$ are same, equation (27) is obtained from equations (25) and (26).

$$
\begin{array}{r}
{\left[\begin{array}{r}
\frac{d i_{\alpha h \mathrm{Va}}}{d t}-\frac{d i_{\alpha h \mathrm{Vb}}}{d t} \\
-\left(\frac{i_{\beta h r \mathrm{Va}}}{d t}-\frac{i_{\beta h r \mathrm{Vb}}}{d t}\right)
\end{array}\right]} \\
=C\left[\begin{array}{l}
l \sin \left(2 \theta-\theta_{\mathrm{Va}}-\theta_{\mathrm{Vb}}\right)+L_{x} \cos \left(2 \theta-\theta_{\mathrm{Va}}-\theta_{\mathrm{Vb}}\right) \\
l \cos \left(2 \theta-\theta_{\mathrm{Va}}-\theta_{\mathrm{Vb}}\right)-L_{x} \sin \left(2 \theta-\theta_{\mathrm{Va}}-\theta_{\mathrm{Vb}}\right)
\end{array}\right] \\
\ldots \ldots \ldots \ldots \ldots \ldots(27)
\end{array}
$$

$f_{\text {sin }}$ and $f_{\text {cos }}$ can be obtained by performing coordinate transformation of equation (27) using $\left(\theta_{\mathrm{Va}}+\theta_{\mathrm{Vb}}\right)$. 


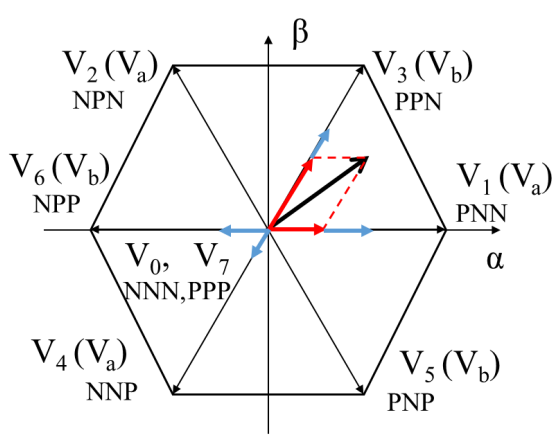

Fig. 5. Modified output vectors

$$
\begin{aligned}
& {\left[\begin{array}{l}
f_{\sin }\left(\frac{d i_{\alpha h}}{d t}, \frac{d i_{\beta h}}{d t}\right) \\
f_{\cos }\left(\frac{d i_{\alpha h}}{d t}, \frac{d i_{\beta h}}{d t}\right)
\end{array}\right]} \\
& =\left[\begin{array}{cc}
\cos \left(\theta_{\mathrm{Va}}+\theta_{\mathrm{Vb}}\right) & -\sin \left(\theta_{\mathrm{Va}}+\theta_{\mathrm{Vb}}\right) \\
\sin \left(\theta_{\mathrm{Va}}+\theta_{\mathrm{Vb}}\right) & \cos \left(\theta_{\mathrm{Va}}+\theta_{\mathrm{Vb}}\right)
\end{array}\right]\left[\begin{array}{c}
\frac{d i_{\alpha h r \mathrm{Va}}}{d t}-\frac{d i_{\alpha h r \mathrm{Vb}}}{d t} \\
-\left(\frac{d i_{\beta h r \mathrm{Va}}}{d t}-\frac{d i_{\beta h r \mathrm{Vb}}}{d t}\right)
\end{array}\right] \\
& =C\left[\begin{array}{l}
l \sin 2 \theta+L_{x} \cos 2 \theta \\
l \cos 2 \theta-L_{x} \sin 2 \theta
\end{array}\right]
\end{aligned}
$$

Equation (28) is equivalent to equations (9) and (10).

Here, it is necessary to modify the PWM output so that the voltage vectors satisfies $\mathrm{V}_{\mathrm{a}}=\mathrm{V}_{\mathrm{b}}$ and are large enough to estimate the position. The PWM modification method is described using the example where the voltage vector reference is between $V_{1}$ and $V_{3}$, as shown in Fig. 5 .

An average voltage vector $\mathbf{e}$ in a sampling period is derived as equation (29). Since $\zeta$ expresses duty ratio, the summation is expressed as (30).

$$
\begin{gathered}
\mathbf{e}=\sum_{n=0}^{7} \zeta_{n} \mathbf{V}_{n} \\
\sum_{n=0}^{7} \zeta_{n}=1 \ldots
\end{gathered}
$$

Generally, PWM utilizes two zero voltage vectors $\left(\mathrm{V}_{0}\right.$ and $\left.\mathrm{V}_{7}\right)$ and two efficient voltage vectors $\left(\mathrm{V}_{\mathrm{a}}\right.$ and $\left.\mathrm{V}_{\mathrm{b}}\right)$ correspond to the output voltage phase. When the reference voltage is small, the duty ratio of $\mathrm{V}_{\mathrm{a}}$ and $\mathrm{V}_{\mathrm{b}}$ also become small. In such case, the current variation in the pulses is too small to estimate the position. In order to amplify the pulse width, not only selected vectors $\mathrm{V}_{\mathrm{a}}$ and $\mathrm{V}_{\mathrm{b}}$ but also their opposite direction vectors are used. In the example of Fig. 5, the selected efficient voltage vectors are $\mathrm{V}_{1}, \mathrm{~V}_{3}, \mathrm{~V}_{6}$, and $\mathrm{V}_{4}$. The reference voltage vector is the black arrow line, the basic voltage vectors are red arrow lines, and the opposite direction voltage vectors are blue arrow lines.

The average voltage vector is then calculated as (31).

$$
\begin{aligned}
\mathbf{e}= & \zeta_{0} \mathbf{V}_{0}+\zeta_{1} \mathbf{V}_{1}+\zeta_{3} \mathbf{V}_{3}+\zeta_{7} \mathbf{V}_{7} \\
= & \zeta_{0}^{\bmod } \mathbf{V}_{0}+\left(\zeta_{1}+\zeta_{6}^{\mathrm{mod}}\right) \mathbf{V}_{1}+\left(\zeta_{3}+\zeta_{4}^{\mathrm{mod}}\right) \mathbf{V}_{3} \\
& +\zeta_{6}^{\mathrm{mod}} \mathbf{V}_{6}+\zeta_{4}^{\mathrm{mod}} \mathbf{V}_{4}+\zeta_{7}^{\mathrm{mod}} \mathbf{V}_{7} \ldots \ldots \ldots \ldots
\end{aligned}
$$

From the assumption utilized in the calculation in equation (27), the output magnitude of $\mathrm{V}_{\mathrm{a}}$ direction vector and $\mathrm{V}_{\mathrm{b}}$

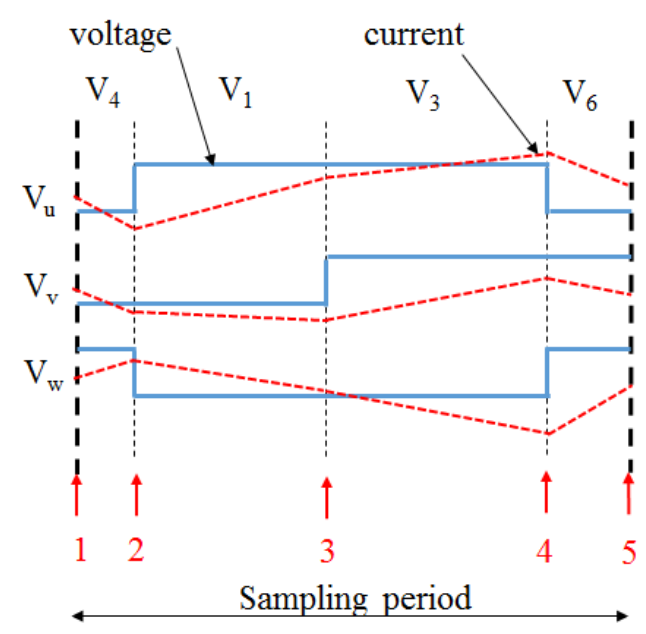

Fig. 6. Modified PWM pattern and current sensing

direction vector are required to be same. The limitation is expressed as (31) with $K_{\zeta}$.

$$
\zeta_{1}+2 \zeta_{6}^{\mathrm{mod}}=\zeta_{3}+2 \zeta_{4}^{\mathrm{mod}}=K_{\zeta} \quad\left(0 \leq K_{\zeta} \leq 0.5\right) \cdots \cdots
$$

The duty ratio of the zero voltage vectors is as follows.

$$
\zeta_{0}^{\text {mod }}+\zeta_{7}^{\text {mod }}=1-2 K_{\zeta}
$$

The larger $K_{\zeta}$ results in the larger pulse width of efficient voltage vectors. In order to achieve large voltage use rate, $K_{\zeta}$ needs to be 0.5 . In such case, the average voltage vector is calculated as (34).

$$
\boldsymbol{e}=\left(\frac{1}{4}+\frac{\zeta_{1}}{2}\right) \boldsymbol{V}_{1}+\left(\frac{1}{4}+\frac{\zeta_{3}}{2}\right) \boldsymbol{V}_{3}+\left(\frac{1}{4}-\frac{\zeta_{1}}{2}\right) \boldsymbol{V}_{6}+\left(\frac{1}{4}-\frac{\zeta_{3}}{2}\right) \boldsymbol{V}_{4}
$$

The output order of selected vectors in one carrier period can be arbitrarily decided. According to the order, the pulse pattern is changed. One example of the patterns is shown in Fig. 6. The number of switching is different depending on the order. As a result, the current variation in a carrier period can be adjusted according to the voltage vector pattern.

In order to obtain the current variation during the voltage vector, the current is sensed every time efficient voltage vectors are switched. Figure 6 shows the timing of the current sensing with arrow. The sensing timing is numbered from 1 to 5 in each sampling period. The current detected at the fifth sampling is utilized as the data for the first sample in the next sampling period. The sampled current is also numbered according to the number of sampling timing. The variation of current is derived as follows.

$$
\begin{aligned}
& \frac{d}{d t}\left[\begin{array}{l}
i_{\alpha h r V a} \\
i_{\beta h r V a}
\end{array}\right]=\frac{1}{t_{c}}\left[\begin{array}{l}
\frac{i_{\alpha 3}-i_{\alpha 2}}{\zeta_{a p}}-\frac{i_{\alpha 5}-i_{\alpha 4}}{\zeta_{a n}} \\
\frac{i_{\beta 3}-i_{\beta 2}}{\zeta_{a p}}-\frac{i_{\beta 5}-i_{\beta 4}}{\zeta_{a n}}
\end{array}\right] \\
& \frac{d}{d t}\left[\begin{array}{c}
i_{\alpha h r V b} \\
i_{\beta h r V b}
\end{array}\right]=\frac{1}{t_{c}}\left[\begin{array}{l}
\frac{i_{\alpha 4}-i_{\alpha 3}}{\zeta_{b p}}-\frac{i_{\alpha 2}-i_{\alpha 1}}{\zeta_{b n}} \\
\frac{i_{\beta 4}-i_{\beta 3}}{\zeta_{b p}}-\frac{i_{\beta 2}-i_{\beta 1}}{\zeta_{b n}}
\end{array}\right]
\end{aligned}
$$

where $t_{c}$ is sampling period, and $\zeta_{a p}, \zeta_{a n}, \zeta_{b p}$ and $\zeta_{b n}$ are the duty of each voltage vectors $\mathrm{V}_{1}, \mathrm{~V}_{6}, \mathrm{~V}_{3}$ and $\mathrm{V}_{4}$ in Fig. 6 . 


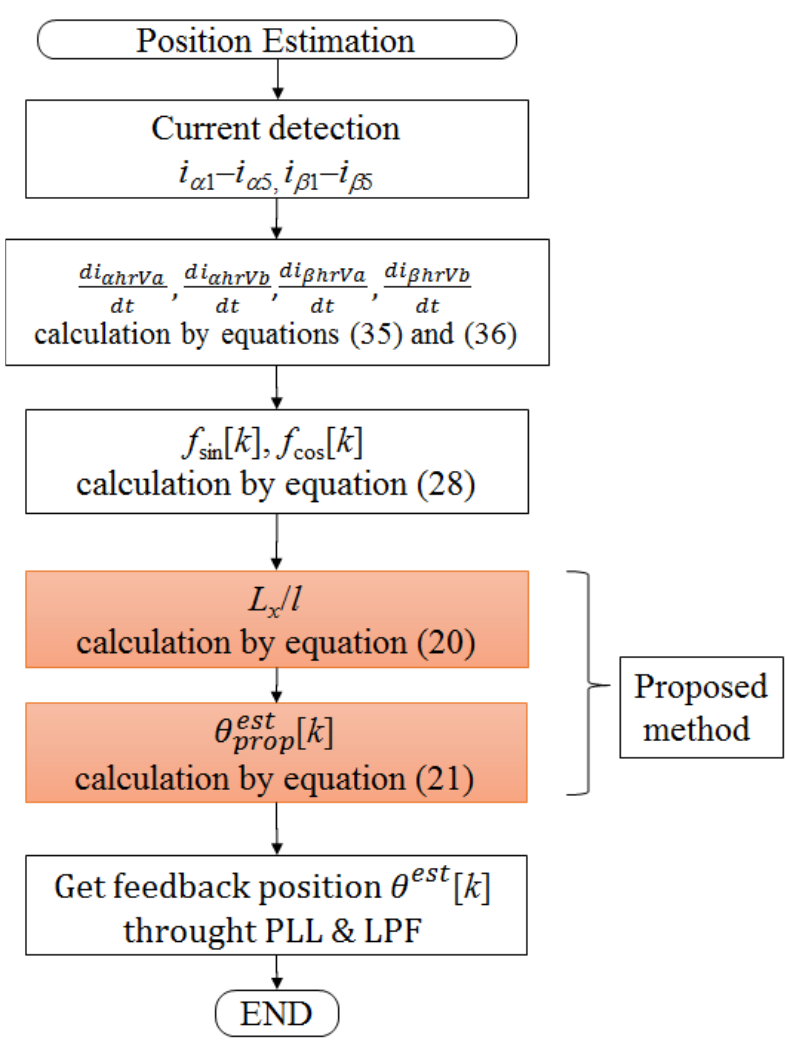

Fig. 7. Position estimation flowchart

From the results obtained by equations (35) and (36), $f_{\text {sin }}$ and $f_{\text {cos }}$ can be calculated by equation (28).

Figure 7 shows the flowchart of the position estimation calculation with the proposed method. By adding the operations of equations (20) and (21) to the conventional operation procedure, the position estimation can be performed in consideration of the dq-axis mutual inductances.

Therefore, the proposed method can be applied to many kinds of position estimation methods with equation (9) and (10) in the procedure of calculating.

\section{Experiments}

In this section, experimental results are shown to prove the effectiveness of the proposed method. The experimental setup, the results of magnetic field analysis of the test motor, and the evaluation results of the position estimation accuracy and the speed control performance are explained.

5.1 Experimental Setup The experimental setup is shown in Fig. 8. The test motor appearance is shown in Fig. 9, and its name plate values are shown in Table 2. Current sensors are connected to A/D converter integrated in Field Programmable Gate Array (FPGA). The A/D converter resolution is 12 bits, and the input is adjusted so that it is about $300 \%$ of the rated current at full scale.

The current informations are sent to Digital Signal Processor (DSP) and position is estimated. By using the estimated position, position or speed control based on FOC is achieved. On the other hand, actual position information is obtained by the rotary encoder attached to the test motor. The encoder detected position is only used for the comparison with estimated values. The magnetic pole position of the encoder is set to the rotor position when $100 \%$ rated current is applied

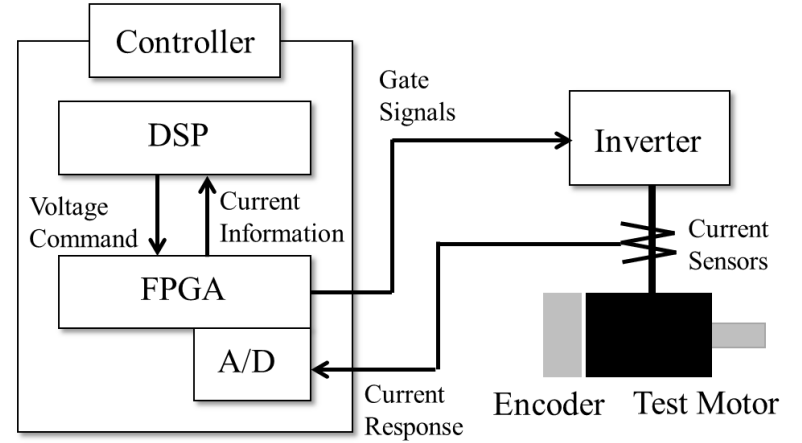

Fig. 8. Experimental setup

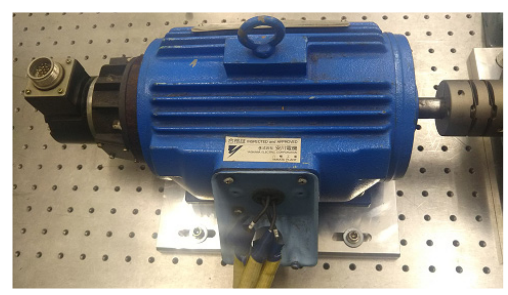

Fig. 9. Test motor

Table 2. Name plate value of the test motor

\begin{tabular}{l|l}
\hline Rated Power & $400[\mathrm{~W}]$ \\
\hline Rated Frequency & $87.5[\mathrm{~Hz}]$ \\
\hline Rated Speed & $1750\left[\mathrm{~min}^{-1}\right]$ \\
\hline Rated Current & $1.8[\mathrm{Arms}]$ \\
\hline Stator Resistance & $2.247[\Omega]$ \\
\hline Nominal Inductance & $L_{d}: 22.47[\mathrm{mH}], L_{q}: 32.5[\mathrm{mH}]$ \\
\hline PM Flux Linkage & $0.2242[\mathrm{~V} \cdot \mathrm{s} / \mathrm{rad}]$ \\
\hline
\end{tabular}

Table 3. Controller parameters

\begin{tabular}{l|l}
\hline Carrier Frequency & $4000[\mathrm{~Hz}]$ \\
\hline Current Control Period & $125[\mu \mathrm{sec}](8000[\mathrm{~Hz}])$ \\
\hline Current Control Bandwidth & $120[\mathrm{~Hz}]$ \\
\hline Speed Control Bandwidth & $5[\mathrm{~Hz}]$ \\
\hline $\begin{array}{l}\text { Attenuation Constant } \\
\text { in Speed Control }\end{array}$ & 0.7 \\
\hline Position Control Bandwidth & $0.83[\mathrm{~Hz}]$ \\
\hline PLL Bandwidth & $60[\mathrm{~Hz}]$ \\
\hline LPF Bandwidth & $40[\mathrm{~Hz}]$ \\
\hline Sensitivity Parameter $a$ & 0.8 \\
\hline
\end{tabular}

at 0 degree of electric angle of the motor. Therefore, the position detected by the encoder may have a slight offset from the actual position.

The controller parameters are shown in Table 3. The proposed method is applied to a sensorless control method based on magnetic saliency described in Section 4. Therefore, the output PWM from the inverter is the modified PWM based on space vector PWM. The sensitivity parameter shown in Table 3 is the value adjusted in advance to reduce the speed ripple when the motor is rotated.

5.2 Magnetic Field Analysis of the Test Motor In IPMSM, the dq-axis cross-coupling components are generated due to magnetic saturation. Normally, magnetic 


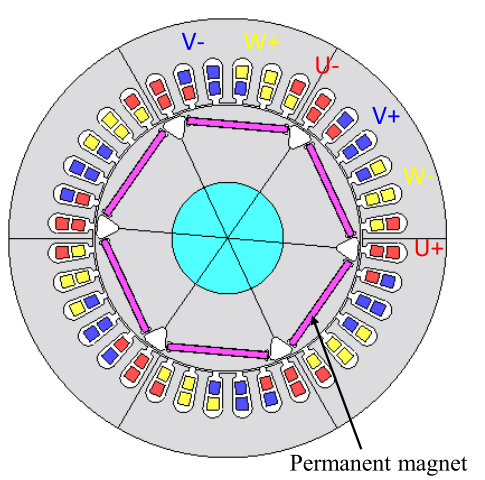

Fig. 10. Cross section of the test motor

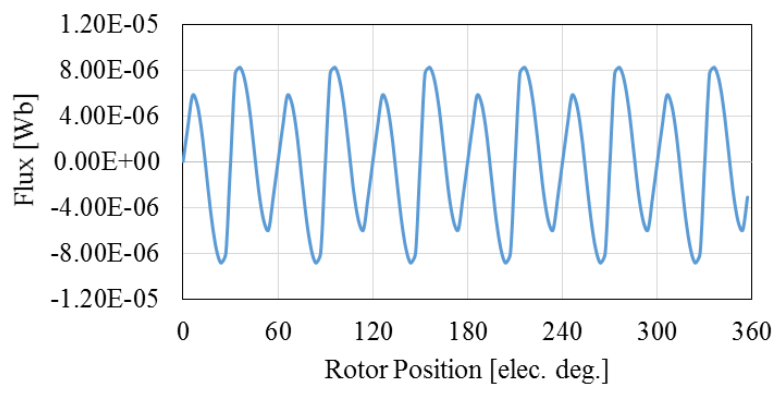

Fig. 11. Analysis of d-axis flux linkage of the test motor

saturation depends on the load current, but in the case of a high-frequency injection sensorless drive, magnetic saturation also occurs due to high-frequency current due to the injection signal. In addition, the magnetic flux linkages contain a little harmonics caused by the rotor shape or the stator slot permeance change. This harmonic component is also contained in the dq-axis cross-coupling component.

Figure 10 shows the cross section of the test motor. The harmonic components due to the structure of the test motor are obtained by magnetic field analysis. Figure 11 shows the result of analysis of the d-axis flux linkage when the permanent-magnet flux $\Phi_{m}=0, i_{q}=i_{d}=0$, and $\Delta i_{d}=0$. The amplitude of the current signal in the analysis is $\Delta i_{q}=0.09 \mathrm{~A}$, which is $5 \%$ of rated current that does not cause magnetic saturation. As shown in Fig. 11, the d-axis flux has a harmonic component depending on the rotor position. Therefore, dqaxis cross-coupling components are generated even under the no load condition. In the saliency-based sensorless control, the harmonic component affects the position estimation accuracy, and increases the speed ripple.

Figure 12(a) shows the test motor inductances under the no-load condition for each rotor position calculated by the following equation based on the magnetic field analysis results ${ }^{(10)}$, Fig. 12(b) shows the expanded waveforms of the mutual inductances $L_{d q}$ and $L_{q d}$, and Fig. 12(c) shows the position error calculated by equation (18) using those inductance value.

$$
\left\{\begin{array}{l}
L_{d}=\left[\psi_{d}\left(i_{d}+\Delta i_{d}, i_{q}, \Phi_{m}\right)-\psi_{d}\left(i_{d}, i_{q}, \Phi_{m}\right)\right] / \Delta i_{d} \\
L_{q}=\left[\psi_{q}\left(i_{d}, i_{q}+\Delta i_{q}, \Phi_{m}\right)-\psi_{q}\left(i_{d}, i_{q}, \Phi_{m}\right)\right] / \Delta i_{q} \\
L_{d q}=\left[\psi_{d}\left(i_{d}, i_{q}+\Delta i_{q}, \Phi_{m}\right)-\psi_{d}\left(i_{d}, i_{q}, \Phi_{m}\right)\right] / \Delta i_{q} \\
L_{q d}=\left[\psi_{q}\left(i_{d}+\Delta i_{d}, i_{q}, \Phi_{m}\right)-\psi_{q}\left(i_{d}, i_{q}, \Phi_{m}\right)\right] / \Delta i_{d}
\end{array}\right.
$$

The mutual inductance variation of the test motor in Fig. 12

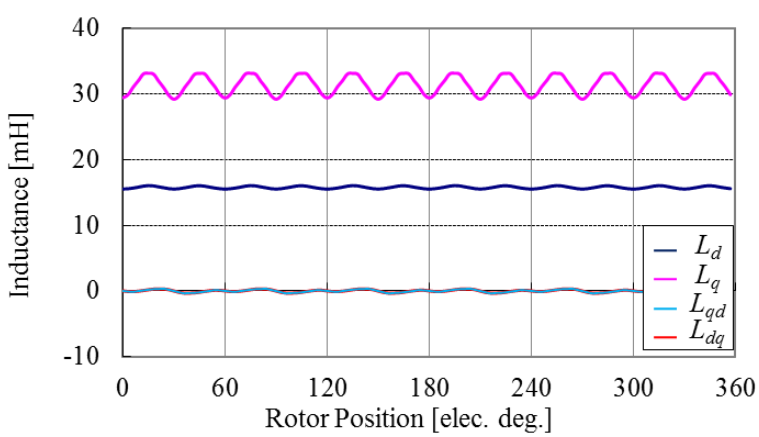

(a) Inductances

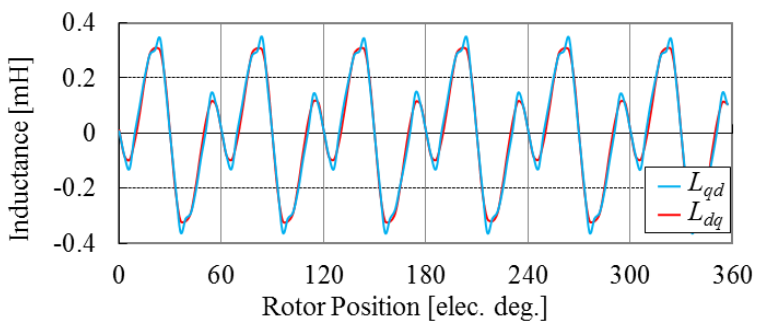

(b) Eexpanded waveforms of the mutual Inductances $L_{d q}, L_{q d}$.

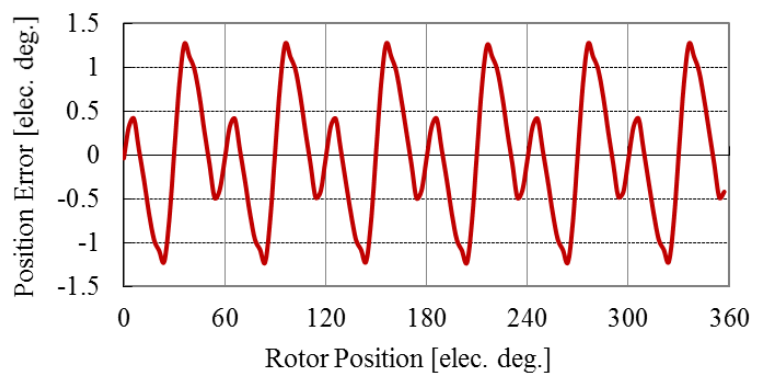

(c) Position Error

Fig. 12. Inductance characteristics and position error (no-load)

is due to the effects of slot and spatial harmonic components of $1 / 12$ and $1 / 6$ electrical angle cycle respectively ${ }^{(14)(15)}$. As shown in Fig. 12(c), the position error includes the effect of these harmonics.

Incidentally, the fact that the inductance of the analysis result is different from the name plate value in Table 2 is due to the difference of the measurement conditions. The inductance measured of the test motor by the LCR meter under conditions close to this analysis is shown below.

$$
\begin{aligned}
& L_{d}: 16.13[\mathrm{mH}] \\
& L_{q}: 27.19[\mathrm{mH}]
\end{aligned}
$$

The analysis value of $L_{d}$ is almost the same as the measured value, but $L_{q}$ in the analysis is larger than the measured value, because the skew applied in the real motor is not taken into account.

5.3 Accuracy of Position Estimation The proposed method compensates the position error of the conventional method. Since the estimated response depends on the applied conventional method, this section evaluates the position accuracy in the steady state of position control.

In order to evaluate the accuracy of position estimation the error between the estimated position and the position detected by encoder is obtained. The control mode switch shown in Fig. 2 is set to position control mode. In the 


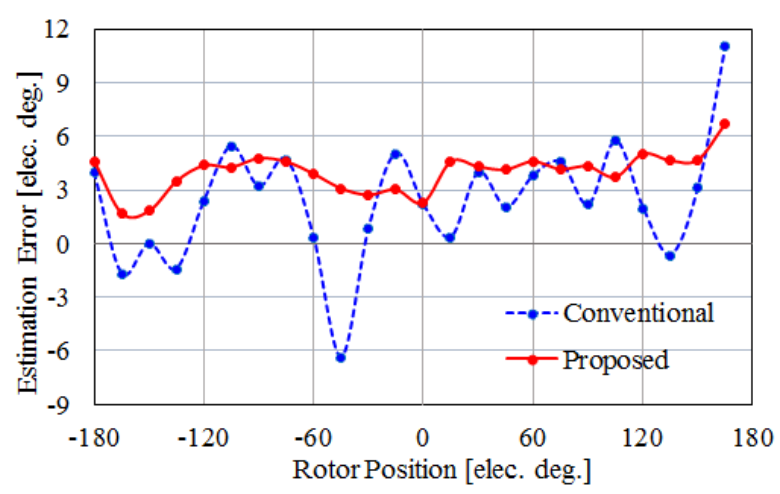

Fig. 13. Estimation error at each electrical angle

Table 4. Comparison of estimation error

\begin{tabular}{l|l|l|l}
\hline \multicolumn{2}{c|}{} & $\begin{array}{l}\text { Proposed } \\
\text { [elec. deg.] }\end{array}$ & $\begin{array}{l}\text { Conventional } \\
\text { [elec. deg.] }\end{array}$ \\
\hline $\begin{array}{l}\text { Position } \\
\text { error }\end{array}$ & Max. & 6.7 & 11.0 \\
\cline { 2 - 4 } & Min. & 1.7 & -6.4 \\
\cline { 2 - 4 } & Average & 4.0 & 2.4 \\
\hline \multicolumn{2}{l}{ Sample standard deviation } & 1.2 & 3.3 \\
\hline
\end{tabular}

experiments, the position controller is a proportional controller, and the speed controller is a proportional-integral controller. Figure 13 shows the error between estimated position and the encoder detected position in the steady state with no load. The drawn data are average values of the position error sampled in $100 \mathrm{~ms}$ with the interval of 15 degree electrical angle. Red dots show the estimated values of the proposed method and blue dots show the estimated values of the conventional method. According to Fig. 13, the spreading of estimation error is decreased. In addition, the max value of the estimation error is also decreased by using proposed method. The quantitative comparison of the result is shown in Table 4.

In Table 4, the average position error are not 0 in both the conventional method and the proposed method. The main reason for this is considered to be the setting error of the magnetic pole position of the encoder. The sample standard deviation of the estimation error of the proposed method is smaller than that of estimation error of the conventional method. The smaller sample standard deviation of the estimation error provides smooth rotation or lower motor noise.

Note that the sample standard deviation $s$ was calculated by the following formula.

$$
s=\sqrt{s^{2}}=\sqrt{\frac{1}{n-1} \sum_{i=1}^{n}\left(X_{i}-\bar{X}\right)^{2}} \ldots \ldots \ldots \ldots \ldots
$$

where, $n$ is the number of samples, $X_{i}$ is the sample value of the position error, and $\bar{X}$ is the average value of the sampled position errors.

5.4 Performance of Speed Control Similarly performance of rotational speed is evaluated by sensorless speed control. The control mode switch shown in Fig. 2 is set to speed control mode. The speed controller is a proportionalintegral controller. Figure 14 and Fig. 15 show the steady state response of speed control to the speed command $10 \%$ of rated speed. The red line shows the estimated angular speed,

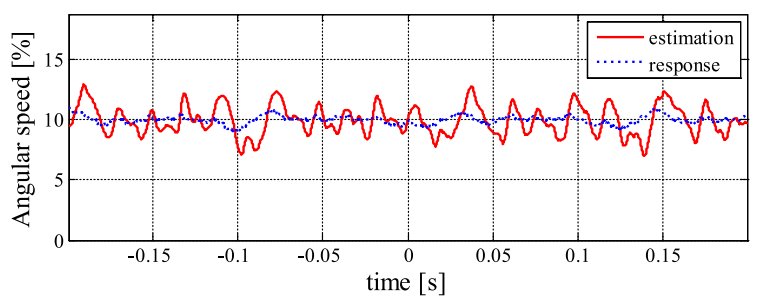

Fig. 14. Estimated speed and actual speed (proposed)

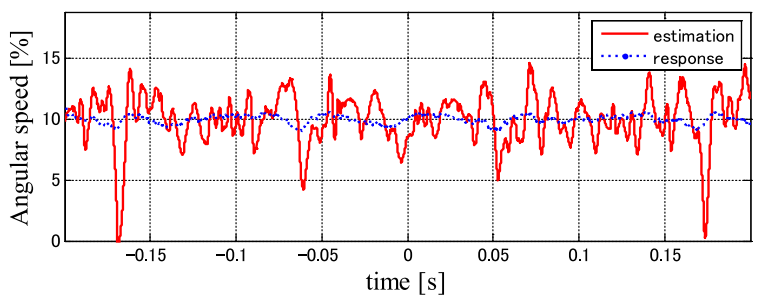

Fig. 15. Estimated speed and actual speed (conventional)

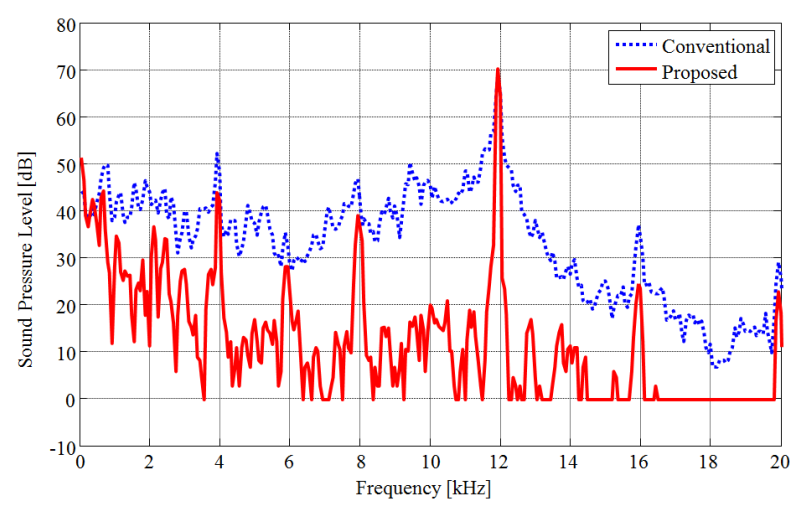

Fig. 16. Sound pressure level of audible noise

and the blue broken line shows the actual speed response. The vibration amplitude of red line in Fig. 14 is smaller than that of red line in Fig. 15. Therefore, the vibration of estimated angular speed can be small by using proposed method. Furthermore, Fig. 16 shows the sound pressure level of the audible noise. The sound pressure levels were measured during the steady-state response of the speed control to the speed command of $10 \%$ of rated speed. The red line shows the sound pressure level of the proposed method, and the blue broken line shows that of the conventional method. By using proposed method, the sound pressure level was decreased from $5 \mathrm{kHz}$ to $10 \mathrm{kHz}$. This frequency band is in the audible range. The noise level reduction is the effect of reducing the speed ripple by compensating the harmonic components of the position estimation.

\section{Conclusion}

In this paper, an improved method of position estimation accuracy was proposed. The method utilizes voltage equation accounting for dq-axis mutual inductances. The proposed method extracts the cross-coupling component from the high-frequency injection signal and uses it to compensate for position estimation, so there is no need to determine the mutual inductance value.

Magnetic field analysis of the test motor showed a change in the mutual inductances depending on the rotor position due 
to slot harmonics. Experimental results show that the position estimation error in the conventional method includes the harmonic components, and that the proposed method can compensate for the position error. Experimental results also show that the proposed method not only improves the estimation accuracy, but also improves the control performance of speed control, and decreases the sound pressure level of the audible noise.

In this paper, the effect of the proposed method on the slot harmonics was confirmed, but the effect on the magnetic saturation and the influence on the response were not sufficiently confirmed. These will be examined in the future.

\section{References}

( 1 ) S.-K. Sul and S. Kim: "Sensorless Control of IPMSM: Past, Present, and Future", IEEJ Journal IA, Vol.1, No.1, pp.15-23 (2012)

( 2 ) M. Schrodl and M. Lambeck: "Statistic Properties of the INFORM-Method in Highly Dynamic Sensorless PM Motor Control Applications Down to Standstill", European Power Electronics and Drives, Vol.13, No.3, pp.22-29 (2003)

( 3 ) J.-I. Ha, K. Ide, T. Sawa, and S.-K. Sul: "Sensorless Position Control and Initial Position Estimation", Conference Record of the 2001 IEEE Industry Applications Conference, 2001, pp.2607-2613 (2001)

( 4 ) S. Ogasawara and H. Akagi: "Implementation and position control performance of a position-sensorless IPM motor drive system based on magnetic saliency", IEEE Trans. Ind. Appl., Vol.34, pp.806-812 (1998)

( 5 ) M. Mamo, K. Ide, M. Sawamura, and J. Oyama: "Novel rotor position extraction based on carrier frequency component method (CFCM) using two reference frames for IPM drives", IEEE Trans. Ind. Electron., Vol.52, No.2, pp.508-514 (2005)

( 6 ) S. Kim, J.-I. Ha, and S.-K. Sul: "PWM Switching Frequency Signal Injection Sensorless Method in IPMSM", IEEE Trans. Ind. Appl., Vol.48, No.5, pp.1576-1587 (2012)

( 7 ) K. Shimamoto, S. Morimoto, and S. Fukumaru: "Sensorless control based on position estimation by switching operation of modified PWM", The 42nd Annual Conference of the IEEE Industrial Electronics Society, pp.2898-2903 (2016)

( 8 ) Y. Lee, Y.-C. Kwon, S.-K. Sul, N.A. Baloch, and S. Morimoto: "Compensation of Position Estimation Error for Precise Position-Sensorless Control of IPMSM Based on High-Frequency Pulsating Voltage Injection", The 9th Annual IEEE Energy Conversion Congress and Exposition, pp.859-864 (2017)

( 9 ) S. Morimoto, K. Shimamoto, and T. Hanamoto: "Realization of High Torque Density Encoderless Servo Drive System", The 45th Annual Conference of the IEEE Industrial Electronics Society, pp.1120-1125 (2019)

(10) K. Yamazaki, M. Kumagai, and T. Fukuoka: "Parameter Determination and Torque Investigation of Interior Permanent Magnet Motors Considering dqAxis Interference Caused by Magnetic Saturation”, IEEJ Trans. Ind. Appl., Vol.133, No.7, pp.747-755 (2013) (In Japanese)

(11) M. Sasaki, K. Shima, K. Ide, H. Koharagi, and M. Takahashi: "The Reactance Calculation for a Permanent Magnet Synchronous Motor Considering Magnetic Saturation”, IEEJ Trans. Ind. Appl., Vol.121, No.7, pp.814-820 (2001) (In Japanese)

(12) Y. Li, Z.Q. Zhu, D. Howe, C.M. Bingham, and D.A. Stone: "Improved RotorPosition Estimation by Signal Injection in Brushless AC Motors, Accounting for Cross-Coupling Magnetic Saturation”, IEEE Trans. on Industry Applications, Vol.45, No.5, pp.1843-1850 (2009)

(13) J. Liu, M. Ma, and L. Li: "A Position Error Compensation way for Sensorless
Linear Motor drive Using High Frequency Injection”, The 16h International Symposium on Electromagnetic Launch Technology (2012)

(14) Y. Kano, T. Kosaka, and N. Matsui: "Torque-Maximizing Design of Concentrated-Winding IPMSM for Saliency-Based Sensorless HEV Drives", IEEJ Trans. Ind. Appl., Vol.135, No.9, pp.929-938 (2015) (In Japanese)

(15) H. Kaimori and K. Akatsu: "Behavior Modeling of Permanent Magnet Synchronous Motors Using Flux Linkages for Coupling with Circuit Simulation”, IEEJ Journal IA., Vol.7, No.1, pp.56-63 (2018)

Shinya Morimoto (Senior Member) received the B.S. degree in Con-

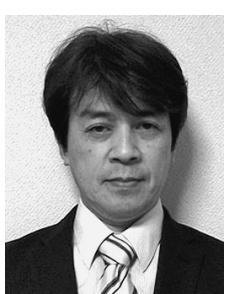
trol Engineering from Kyushu Institute of Technology, Kitakyushu City, Japan, in 1990, and is presently a Chief Manager of the EV Powertrain System Department at the Corporate Technology Division in Yaskawa Electric Corporation. He has worked on development of motor-drive control and power conversion technology. He is a member of SICE Japan, ISCIE Japan and IEEE IAS.

Keita Shimamoto (Member) received the B.S. degree in system de-

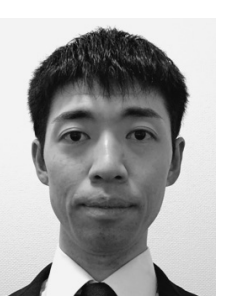
sign engineering and the M.S. degree in integrated design engineering from Keio University, Yokohama, Japan, in 2012 and 2014 respectively. He is presently at the Tsukuba Research Laboratory in Yaskawa Electric Corporation. He has worked on research of motion control technology. He is a member of IEEE IES.

Masanobu Kakihara (Member) received B.S. and M.S. degrees

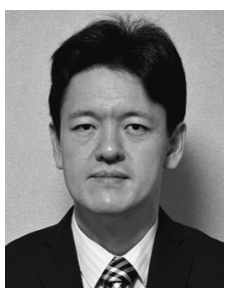
in electrical engineering from Kyushu University, Fukuoka, Japan, in 2003 and 2005, respectively. In 2005, he joined Yaskawa Electric Corporation, Kitakyushu, Japan, where he is currently a Member of the Motor Actuator Development Dept., Corporate R\&D Center. His current research interests include the design and development of servo motors and actuators.

Tsuyoshi Hanamoto (Member) received the B.S. and M.S. degrees

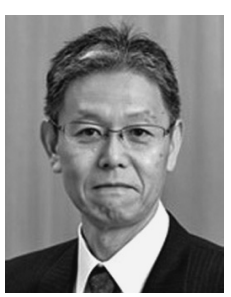
from Kyushu Institute of Technology, Kitakyushu, Japan, in 1984 and 1986, respectively. In 1986, he joined the Kobe Works of Kobe Steel, Ltd., Japan. In 1990, he joined the Center for Cooperative Research, Kyushu Institute of Technology. From 1997 to 2000, he was with the Department of Electrical Engineering, Kyushu Institute of Technology. Since April 2000, he has been with the Graduate School of Life Science and Systems Engineering, Kyushu Institute of Technology, where he is currently a Professor. His research interests include motor control and power conversion. 